\title{
The impact of comorbidity and stage on ovarian cancer mortality: A nationwide Danish cohort study Mette S Tetsche ${ }^{* \dagger 1,2}$, Claus Dethlefsen ${ }^{\dagger 4}$, Lars Pedersen ${ }^{\dagger 1}$, Henrik T Sorensen ${ }^{\dagger 1,3}$ and Mette Norgaard ${ }^{\dagger 1}$
}

Address: ${ }^{1}$ Department of Clinical Epidemiology, Aarhus University Hospital, 8000 Aarhus, Denmark, ${ }^{2}$ Department of Gynecology, Aalborg Hospital, Aarhus University Hospital, 9000 Aalborg, Denmark, ${ }^{3}$ Department of Epidemiology, Boston University School of Public Health, Boston, MA 02118, USA and ${ }^{4}$ Center for Cardiovascular Research, Aalborg Hospital, Aarhus University Hospital, 9000 Aalborg, Denmark

Email: Mette S Tetsche* - mst@rn.dk; Claus Dethlefsen - cld@rn.dk; Lars Pedersen - lap@dce.au.dk; Henrik T Sorensen - hts@dce.au.dk; Mette Norgaard - m.noergaard@rn.dk

* Corresponding author †Equal contributors

Published: 29 January 2008

BMC Cancer 2008, 8:31 doi:|0.||86/|47|-2407-8-3|
Received: 4 October 2007

Accepted: 29 January 2008

This article is available from: http://www.biomedcentral.com/I47I-2407/8/3I

(C) 2008 Tetsche et al; licensee BioMed Central Ltd.

This is an Open Access article distributed under the terms of the Creative Commons Attribution License (http://creativecommons.org/licenses/by/2.0), which permits unrestricted use, distribution, and reproduction in any medium, provided the original work is properly cited.

\begin{abstract}
Background: The incidence of ovarian cancer increases sharply with age, and many elderly patients have coexisting diseases. If patients with comorbidities are diagnosed with advanced stages, this would explain the poor survival observed among ovarian cancer patients with severe comorbidity. Our aims were to examine the prevalence of comorbidity according to stage of cancer at diagnosis, to estimate the impact of comorbidity on survival, and to examine whether the impact of comorbidity on survival varies by stage.
\end{abstract}

Methods: From the Danish Cancer Registry we identified 5,213 patients ( $>15$ years old) with ovarian cancer diagnosed from 1995 to 2003. We obtained information on comorbidities from the Danish National Hospital Discharge Registry. Vital status was determined through linkage to the Civil Registration System. We estimated the prevalence of comorbidity by stage and computed absolute survival and relative mortality rate ratios (MRRs) by comorbidity level (Charlson Index score $0,1-2,3+$ ), using patients with Charlson Index score 0 as the reference group. We then stratified by stage and computed the absolute survival and MRRs according to comorbidity level, using patients with Charlson score 0 and localized tumour/FIGO I as the reference group. We adjusted for age and calendar time.

Results: Comorbidity was more common among patients with an advanced stage of cancer. Oneand five-year survival was higher in patients without comorbidity than in patients with registered comorbidity. After adjustment for age and calendar time, one-year MRRs declined from I.8 to I.4 and from 2.7 to 2.0, for patients with Charlson scores I-2 and 3+, respectively. After adjustment for stage, the MRRs further declined to I.3 and I.8, respectively. Five-year MRRs declined similarly after adjustment for age, calendar time, and stage. The impact of severe comorbidity on mortality varied by stage, particularly among patients with tumours with regional spread/FIGO-stages II and III.

Conclusion: The presence of severe comorbidity was associated with an advanced stage of ovarian cancer. Mortality was higher among patients with comorbidities and the impact of comorbidity varied by stage. 


\section{Background}

Ovarian cancer is the leading cause of death from gynaecological cancer in western countries. It has a poor prognosis, with five-year survival rates ranging from $26 \%$ to $51 \%$ in Europe [1]. As the incidence of ovarian cancer increases sharply with age, many patients have one or more other chronic diseases, i.e., comorbidities [2,3]. Comorbidity is an important predictor of prognosis in patients with chronic diseases, including cancer $[4,5]$. Among women with ovarian cancer, the presence of comorbidity may substantially influence the diagnostic work-up, alter treatment efficacy, and affect survival.

Few studies to date have examined the impact of comorbidity on ovarian cancer survival [6-11]. The presence of comorbidity at time of diagnosis was found to have a negative impact on prognosis and survival in two studies $[7,9]$, but not in others $[6,8,10,11]$. Based on hospital discharge registry data, we recently reported that one-year mortality in a Danish regional study was twice as high in ovarian cancer patients with severe comorbidity as in those without recorded comorbidity [12]. However, the study was limited by lack of information on the stage of cancer and by our inability to exclude patients with borderline tumours.

The presence of comorbidities may influence stage at diagnosis, which in turn is a strong predictor of survival in ovarian cancer patients. It is possible that patients with comorbidities experience delay in diagnosis, resulting in a more advanced cancer stage. We thus hypothesized that poorer survival among ovarian cancer patients with severe comorbidity, compared with those without comorbid conditions, may be explained by a higher prevalence of advanced cancer at diagnosis.

The Danish Cancer Registry records stage information on incident ovarian cancers. Using data from this Registry, we conducted a nationwide study to determine the prevalence of comorbidity by stage of ovarian cancer, to estimate the impact of comorbidity on survival and mortality, controlling for cancer stage, and to examine whether the effect of comorbidity on ovarian cancer mortality varies by cancer stage at diagnosis.

\section{Methods}

We conducted this nationwide study in Denmark, which has approximately 5.4 million inhabitants, from January 1, 1995 to December 31, 2005. The entire Danish population has free access to tax-supported medical care, including hospitalization. There is practically no private inpatient ovarian cancer treatment available. In 2004, surgery for ovarian cancer took place in 52 hospital departments [13], five of which were gynecologic oncologic centers. Thus, treatment of this cancer is quite decentralized in the Danish setting.

\section{Identification of ovarian cancer patients The Danish Cancer Registry}

The Danish Cancer Registry has kept records of all patients in Denmark with malignant neoplasms since 1943 [14]. The cancer stage at the time of diagnosis is reported to the Danish Cancer Registry as either localized tumour, tumour with regional spread, tumour with distant metastases or, alternatively, by stage according to the FIGO classification [15]. For tumours categorized as regional spread, we were unable to determine whether it was a FIGO-stage II or FIGO-stage III tumour, therefore we used the scheme of Kjaerby-Thygesen et al. to categorize ovarian cancer cases into four groups: (a) localized tumours/ FIGO-stage I tumours; (b) tumours with regional spread/ FIGO-stage II and III tumours; (c) tumours with distant metastases/FIGO-stage IV tumours (also referred to as "advanced stage" below); and (d) tumours with unspecified stage [16]. The Cancer Registry provided dichotomized data on cancer treatment within the first four months following diagnosis, such as surgery (yes/no), chemotherapy (yes/no), radiation (yes/no), and other (yes/no) [17]. The Registry also contains information on histological types.

We searched the Danish Cancer Registry for patients with a first-time ovarian cancer diagnosis [International Classification of Diseases (ICD), $7^{\text {th }}$ revision, codes 175.0, 175.1, 175.2, 175.3, 375.0, 475.0, or 875.0] [18] between 1 January, 1995 and 31 December, 2003. We omitted children since ovarian cancer seldom occurs in childhood and often has a different clinical picture than in adults. Eight patients younger than 15 years thus were excluded from the analysis.

\section{Data on comorbidity}

\section{The Danish Hospital Discharge Registry}

The Danish National Hospital Discharge Registry [19] contains information on all patients discharged from non-psychiatric hospitals in Denmark since 1977. Data on outpatient visits have been included since 1995 [20]. Information initially is collected in county-specific hospital discharge registries immediately upon discharge, and then is transferred to the National Registry. Data in this Registry, which covers more than $99 \%$ of all non-psychiatric discharges nationwide [19], are used routinely to monitor hospital admissions and discharges, waiting lists, operations, and treatment. Records include civil registration number (CPR), dates of admission and discharge, surgical procedure(s) performed, and up to 20 discharge diagnoses, which are classified by physicians according to the Danish version of the International Classification of 
Diseases (ICD) (8th revision until the end of 1993 and 10th revision thereafter) [19].

For each patient with ovarian cancer identified from the Danish Cancer Registry, we extracted all discharge diagnoses documented in the Hospital Discharge Registry between January 1, 1977 and the date of ovarian cancer diagnosis. We obtained 18 to 26 years of hospitalization history for each patient, depending on date of diagnosis, and used this information to compute the Charlson Comorbidity Index [4]. This Index is a weighted index of the number and the seriousness of comorbid diseases [21], which has been widely used in studies of cancer patients [5,22-25]. Because ovarian cancer defined our study cohort, we excluded this diagnosis from Index calculations. The comorbid conditions in the 5,213 ovarian cancer patients are shown in Table 1.

Patients were categorized in three groups according to their Charlson Index comorbidity score: score 0 (no recorded comorbidity), score 1-2 (moderate comorbidity), and score 3 or more (severe comorbidity).

\section{Record linkage}

Since 1968, a unique 10-digit civil registration number has been assigned to each Danish resident by the Central Office of Civil Registration. Use of this number permits data linkage between registries. The Civil Registration System is updated daily, and contains information on vital status, date of death, and the residence of all Danish residents [26]. We used information from this Registry to follow patients until death, emigration, or 31 December

Table I: Comorbid conditions in the 5,2 13 ovarian cancer patients

\begin{tabular}{lcc}
\hline Conditions & Total (\%) & Charlson score \\
\hline Myocardial infarct & $113(2.2 \%)$ & $\mathrm{I}$ \\
Congestive heart failure & $117(2.2 \%)$ & $\mathrm{I}$ \\
Peripheral vascular disease & $112(2.2 \%)$ & $\mathrm{I}$ \\
Cerebrovascular disease & $229(4.4 \%)$ & $\mathrm{I}$ \\
Dementia & $17(0.3 \%)$ & $\mathrm{I}$ \\
Chronic pulmonary disease & $232(4.5 \%)$ & 1 \\
Connective tissue disease & $137(2.6 \%)$ & 1 \\
Ulcer disease & $180(3.5 \%)$ & 1 \\
Mild liver disease & $47(0.9 \%)$ & 1 \\
Diabetes type I or type 2 & $124(2.4 \%)$ & 1 \\
Hemiplegia & $8(0.2 \%)$ & 2 \\
Moderate or severe renal disease & $39(0.8 \%)$ & 2 \\
Diabetes with end organ damage type & $50(1.0 \%)$ & 2 \\
I or type 2 & & \\
Any tumour (not ovarian cancer) & $490(9.4 \%)$ & 2 \\
Leukemia & $9(0.2 \%)$ & 2 \\
Lymphoma & $13(0.3 \%)$ & 2 \\
Moderate or severe liver disease & $11(0.2 \%)$ & 3 \\
Metastatic solid tumour & $160(3.1 \%)$ & 6 \\
AIDS & 0 & 6 \\
\hline & & \\
\hline & &
\end{tabular}

2005, whichever came first, and to compute all-cause mortality.

\section{Statistical analyses}

Stage of cancer associated with comorbidity

We constructed contingency tables of cancer stage and comorbidity level. Using Mantel-Haenszel methods [27], we then computed age-adjusted prevalence ratios to compare the prevalence of distant metastases/FIGO IV among patients with comorbidity with that among patients without comorbidity.

\section{Comorbidity, stage of cancer and survival}

We computed Kaplan-Meier survival curves for each Charlson comorbidity group and cancer stage (localized/ FIGO-stage I tumours, tumours with regional spread/ FIGO-stage II and III tumours, and tumours with distant metastases/FIGO-stage IV tumours).

Using Cox proportional hazards regression analysis, we computed one- and five-year crude and adjusted hazard ratios as a measure of mortality rate ratios [28]. Patients with no registered comorbidity served as the reference group. We adjusted first for age and calendar time (3-year calendar periods) and then for cancer stage.

Design variables were created for the 12 combinations of stage and comorbidity. For each stratum we computed one- and five-year survival using Kaplan-Meier product limit methods [29], absolute survival was defined as the proportion of the patients who were still alive one- or fiveyears after diagnosis. Cox proportional hazards regression analysis was used to compare mortality rates. Patients with localized tumours/FIGO-stage I tumours and no registered comorbidity served as the reference group. We adjusted for age and year of diagnosis (3-year calendar periods).

In addition, the analyses were repeated adjusting for treatment. We also conducted analyses restricted to ovarian cancer patients who had received either surgery, chemotherapy or both within the first four months after diagnosis.

We verified the assumption of proportional hazards in the Cox model graphically. Estimates are provided with their corresponding 95\% Confidence Intervals (95\% CI). Analyses were performed using STATA version 9.2 (Stata Corporation, College Station, Tx, USA).

\section{Results \\ Descriptive data}

We identified 5,213 patients above 15 years of age diagnosed with ovarian cancer from 1995 to 2003. Of these, $3,727(72 \%)$ had no comorbidity recorded in the 
National Hospital Discharge Registry, 1,116 (21\%) had Charlson score 1-2, and 370 (7\%) had Charlson score 3+ (Table 2). The median age was 62 years in patients without comorbidity, 70 years in patients with Charlson score $1-2$, and 71 years in patients with Charlson score 3+. Patients without comorbidity were more frequently treated with surgery/chemotherapy (47\%) than patients with severe comorbidity (27\%) (Table 2).

An association between comorbidity and advanced stage, for whom information on stage of disease was available, was found only among patients with severe comorbidity. The age-adjusted prevalence ratio was 1.0 (95\% CI, 0.9-1.1) among patients with moderate comorbidity. Among patients with severe comorbidity, $42 \%$ had distant metastases/FIGO IV, compared to $28 \%$ of patients without comorbidity (age-adjusted prevalence ratio $=1.3$, 95\% CI, 1.1-1.5).

\section{Comorbidity survival and mortality}

Estimates of one- and five-year survival and MRR by level of comorbidity are shown in Table 3. One- and five-year survival was higher in patients without comorbidity than in patients with registered comorbidity. After adjustment for age and calendar time, one-year MRRs declined from 1.8 to 1.4 for patients with Charlson score $1-2$ and from 2.7 to 2.0 for patients with Charlson score $3+$. When adjustment for stage was added to the model, the MRRs further declined to 1.3 for patients with Charlson score 1-2 and 1.8 for patients with Charlson score $3+$. Five-year MRRs declined similarly after adjusting for age, calendar time, and stage.

\section{Comorbidity, stage of cancer and survival}

Figures 1, 2, 3 show survival curves for patients with ovarian cancer by level of comorbidity at time of diagnosis. For all stages, survival was higher in patients without comorbidity than in patients with comorbidity. One- and five-year survivals are shown in Tables 4 and 5 .

For all stages, we found higher crude MRRs among patients with comorbidity compared with patients without comorbidity. While MRRs declined after adjustment for age and calendar time, they remained higher among patients with comorbidity (Tables 4 and 5).

Table 2: Characteristics of ovarian cancer patients, $N=5,213$.

\begin{tabular}{|c|c|c|c|}
\hline & \multicolumn{3}{|c|}{ Charlson Comorbidity score } \\
\hline & Comorbidity 0 & Comorbidity I-2 & Comorbidity $3+$ \\
\hline Number & 3,727 (72\%) & $1,116(21 \%)$ & $370(7 \%)$ \\
\hline Median age, years & 62 & 70 & 71 \\
\hline ( $25 \%-75 \%$ percentile) & $(52-72)$ & $(59-77)$ & $(62-78)$ \\
\hline \multicolumn{4}{|l|}{ Age groups } \\
\hline$<50$ years & $726(19 \%)$ & $96(9 \%)$ & $21(6 \%)$ \\
\hline $50-69$ years & $\mathrm{I}, 897(5 \mathrm{I} \%)$ & $474(42 \%)$ & $153(41 \%)$ \\
\hline 70-89 years & $1,079(29 \%)$ & $521(47 \%)$ & $188(5 \mid \%)$ \\
\hline $90+$ years & $25(<1 \%)$ & $25(2 \%)$ & $8(2 \%)$ \\
\hline \multicolumn{4}{|l|}{ Year of diagnosis } \\
\hline $1995-1997$ & $\mathrm{I}, 278(74 \%)$ & $336(19 \%)$ & $119(7 \%)$ \\
\hline $1998-2000$ & $1,300(71 \%)$ & $406(22 \%)$ & $119(7 \%)$ \\
\hline $200 \mathrm{I}-2003$ & $1,149(69 \%)$ & $374(23 \%)$ & $132(8 \%)$ \\
\hline \multicolumn{4}{|l|}{ Extent of cancer } \\
\hline Localized/FIGO I & $774(21 \%)$ & $184(16 \%)$ & $37(10 \%)$ \\
\hline Regional/FIGO II and III & $\mathrm{I}, 757(47 \%)$ & 492 (44\%) & $157(42 \%)$ \\
\hline Distant metastases/FIGO IV & $1,002(27 \%)$ & $338(30 \%)$ & 140 (38\%) \\
\hline Unspecified/missing & $194(5 \%)$ & $102(9 \%)$ & $36(10 \%)$ \\
\hline \multicolumn{4}{|l|}{ Histological type } \\
\hline Epithelial & $3,422(92 \%)$ & $985(88 \%)$ & $322(87 \%)$ \\
\hline Non-epithelial & $305(8 \%)$ & $131(12 \%)$ & $48(13 \%)$ \\
\hline \multicolumn{4}{|l|}{ Treatment } \\
\hline Surgery/Chemotherapy & $\mathrm{I}, 753(47 \%)$ & $378(34 \%)$ & I0I (27\%) \\
\hline Surgery & $1,416(38 \%)$ & 442 (40\%) & $132(36 \%)$ \\
\hline Chemotherapy & $230(6 \%)$ & $94(8 \%)$ & $32(9 \%)$ \\
\hline Other* & $12(<1 \%)$ & $9(<1 \%)$ & $6(2 \%)$ \\
\hline No/Symptomatic & $285(8 \%)$ & $178(16 \%)$ & $94(25 \%)$ \\
\hline Missing & $31(<1 \%)$ & $15(1 \%)$ & $5(1 \%)$ \\
\hline
\end{tabular}

*Hormonal treatment, radiation, other not specified 
Table 3: One- and five-year mortality rate ratios (MRRs) by level of comorbidity. The corresponding $95 \%$ confidence interval is given in parentheses.

\begin{tabular}{|c|c|c|c|}
\hline & \multicolumn{3}{|c|}{ Charlson Comorbidity score } \\
\hline & $\mathbf{0}$ & $1-2$ & $3+$ \\
\hline $\mathrm{N}(\%)$ & $3,727(72 \%)$ & $1,116(2 \mid \%)$ & $370(7 \%)$ \\
\hline \multicolumn{4}{|l|}{ I-year } \\
\hline Survival in \% & $73(7 \mid-74)$ & $58(55-60)$ & $44(39-49)$ \\
\hline MRR & I (ref.) & $1.8(1.6-2.0)$ & $2.7(2.3-3.1)$ \\
\hline Adj. MRR* & I (ref.) & $1.4(1.2-1.5)$ & $2.0(1.7-2.3)$ \\
\hline Adj. MRR*** & I (ref.) & $1.3(1.2-1.5)$ & $1.8(1.6-2.1)$ \\
\hline \multicolumn{4}{|l|}{ 5-year } \\
\hline Survival in \% & $37(36-39)$ & $24(2 \mid-27)$ & $12(8-16)$ \\
\hline MRR & I (ref.) & $1.5(1.4-1.6)$ & $2.3(2.1-2.6)$ \\
\hline Adj. MRR* & I (ref.) & $1.3(1.2-1.4)$ & $1.8(1.6-2.1)$ \\
\hline Adj. MRR*** & I (ref.) & $1.2(1.1-1.4)$ & $1.7(1.5-1.9)$ \\
\hline
\end{tabular}

*Adjusted for age and calendar time.

**Adjusted for age, calendar time and stage.

We found a varying effect of severe comorbidity on oneyear mortality according to stage of ovarian cancer. Tumours with regional spread/FIGO stage II and III further increased the impact of severe comorbidity on mortality (Table 4). Compared with patients with no registered comorbidity and localized tumour/FIGO I, patients with severe comorbidity and this cancer stage had an adjusted MRR of 2.7, patients with no registered comorbidity and tumours with regional spread/FIGO stage II and III had an adjusted MRR of 4.8 , and patients with severe comorbidity and tumours with regional spread/FIGO stage II and III had an adjusted MRR of 12.3. Although not as pronounced, a similar variation in the effect of severe comorbidity by stage was seen in five-year

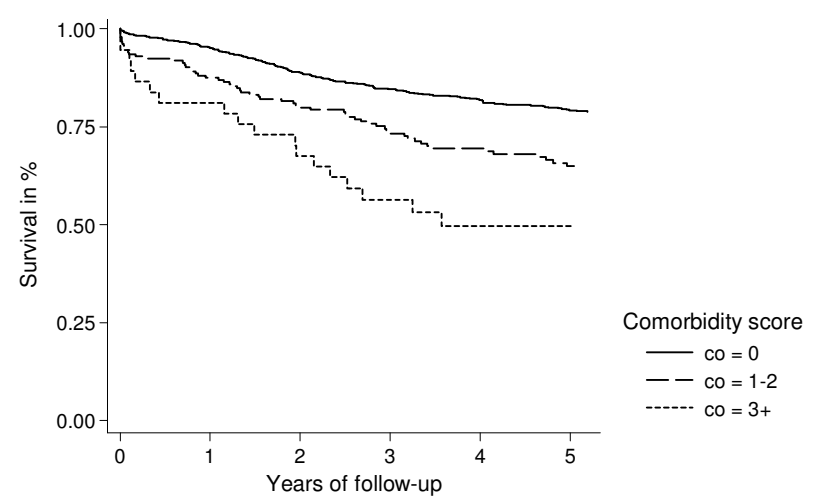

\section{Figure I}

Kaplan-Meier survival curves for ovarian cancer patients with localized tumour/FIGO stage I, according to presence of comorbidity at time of diagnosis.

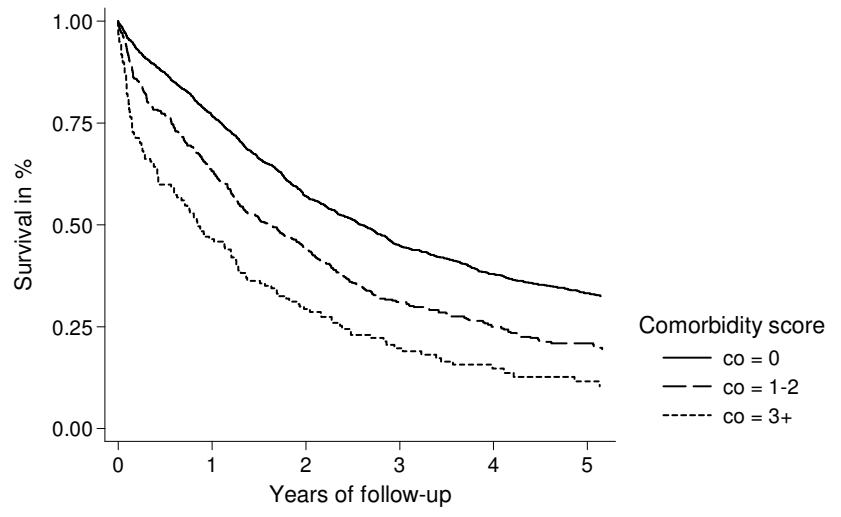

Figure 2

Kaplan-Meier survival curves for ovarian cancer patients with regional spread/FIGO stage II and III, according to presence of comorbidity at time of diagnosis.

mortality (Table 5). The impact of moderate comorbidity on one- and five-year mortality varied only slightly by stage.

Including treatment in the analysis did not remove the association between severe comorbidity and mortality (data not shown), except for tumours with distant metastases/FIGO-stage IV.

Analyses restricted to patients who received either surgery, chemotherapy or both yielded results similar to analyses including all ovarian cancer patients (data not shown).

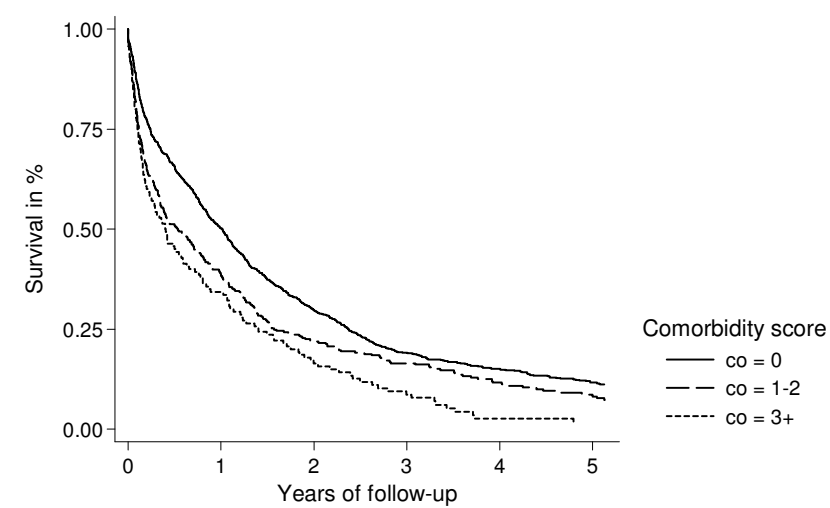

Figure 3

Kaplan-Meier survival curves for ovarian cancer patients with distant metastases/FIGO stage IV, according to presence of comorbidity at time of diagnosis. 
Table 4: One-year survival and one-year mortality rate ratio (MRR) by level of comorbidity according to the stage of cancer and adjusted for age and calendar time.

\begin{tabular}{|c|c|c|c|}
\hline & \multicolumn{3}{|c|}{ Charlson Comorbidity score } \\
\hline & 0 & $1-2$ & $3+$ \\
\hline \multicolumn{4}{|c|}{ Localized tumour/FIGO-stage I } \\
\hline Number & 774 & 184 & 37 \\
\hline Median age, years & 56 & 67 & 72 \\
\hline Survival in \% & $95(93-97)$ & $88(82-92)$ & $81(64-91)$ \\
\hline Crude MRR & I (ref.) & $2.8(1.6-4.6)$ & $4.5(2.0-10.0)$ \\
\hline Adj. MRR & I (ref.) & $2.1(1.2-3.5)$ & $2.7(1.2-6.2)$ \\
\hline \multicolumn{4}{|c|}{ Regional spread/FIGO-stage II, III } \\
\hline Number & ।,757 & 492 & 157 \\
\hline Median age, years & 62 & 68 & 69 \\
\hline Survival in \% & 77 (75-79) & $63(59-67)$ & $47(39-54)$ \\
\hline Crude MRR & $5.3(3.8-7.4)$ & $9.2(6.5-13.2)$ & $16.0(10.9-23.6)$ \\
\hline Adj. MRR & $4.8(3.5-6.8)$ & $7.1(5.0-10.1)$ & $12.3(8.3-18.1)$ \\
\hline \multicolumn{4}{|c|}{ Distant metastases/FIGO-stage IV } \\
\hline Number & 1,002 & 338 & 140 \\
\hline Median age, years & 66 & 71 & 71 \\
\hline Survival in \% & $50(47-53)$ & $38(33-44)$ & $34(27-42)$ \\
\hline Crude MRR & $14.1(10.1-19.7)$ & $20.4(14.4-28.9)$ & $23.4(16.0-34.2)$ \\
\hline Adj. MRR & $11.6(8.3-16.2)$ & $13.9(9.8-19.8)$ & $15.7(10.7-23.1)$ \\
\hline \multicolumn{4}{|l|}{ Unspecified } \\
\hline Number & 194 & 102 & 36 \\
\hline Median age, years & 66 & 75 & 71 \\
\hline Survival in \% & $62(55-68)$ & $40(31-50)$ & 33 (19-49) \\
\hline Crude MRR & $10.1(6.8-15.0)$ & $19.2(12.8-28.9)$ & $25.0(|5.0-4| .8)$ \\
\hline Adj. MRR & $8.1(5.5-12.1)$ & $11.2(7.4-16.8)$ & $15.3(9.1-25.7)$ \\
\hline
\end{tabular}

The reference group was patients with localized tumour/FIGO I and no registered comorbidity. The corresponding $95 \%$ confidence interval is given in parentheses.

Table 5: Five-year survival and five-year all-cause mortality rate ratio (MRR) by level of comorbidity according to the FIGO-stages and adjusted for age and calendar time.

\begin{tabular}{|c|c|c|c|}
\hline & \multicolumn{3}{|c|}{ Charlson Comorbidity score } \\
\hline & 0 & $1-2$ & $3+$ \\
\hline \multicolumn{4}{|c|}{ Localized tumour/FIGO-stage I } \\
\hline Survival in \% & $79(76-82)$ & $65(57-72)$ & $50(32-65)$ \\
\hline Crude MRR & I (ref.) & $1.8(1.4-2.5)$ & $3.2(2.0-5.3)$ \\
\hline Adj. MRR & I (ref.) & $1.6(1.2-2.1)$ & $2.3(1.4-3.8)$ \\
\hline \multicolumn{4}{|c|}{ Regional spread/FIGO-stage II, III } \\
\hline Survival in \% & $33(3 \mid-36)$ & $21(17-25)$ & $12(7-18)$ \\
\hline Crude MRR & $4.5(3.8-5.4)$ & $6.7(5.6-8.1)$ & $10.1(8.0-12.7)$ \\
\hline Adj. MRR & $4.4(3.7-5.2)$ & $5.7(4.7-6.9)$ & $8.6(6.8-10.9)$ \\
\hline \multicolumn{4}{|c|}{ Distant metastases/FIGO-stage IV } \\
\hline Survival in \% & $12(10-14)$ & $8(5-12)$ & $2(0.4-6)$ \\
\hline Crude MRR & $9.7(8.1-11.5)$ & $12.4(10.2-15.0)$ & $15.9(12.6-20.0)$ \\
\hline Adj. MRR & $8.9(7.5-10.6)$ & $9.7(8.0-11.8)$ & $12.1(9.6-15.4)$ \\
\hline \multicolumn{4}{|l|}{ Unspecified } \\
\hline Survival in \% & $36(29-43)$ & $17(10-26)$ & $14(5-29)$ \\
\hline Crude MRR & $5.1(4.0-6.5)$ & $9.8(7.5-12.8)$ & $11.8(8.0-17.5)$ \\
\hline Adj. MRR & $4.6(3.6-5.8)$ & $6.8(5.2-8.9)$ & $8.0(5.4-12.0)$ \\
\hline
\end{tabular}

The reference group was patients with localized tumour/FIGO I and no registered comorbidity. The corresponding $95 \%$ confidence interval is given in parentheses. 


\section{Discussion}

In this population-based nationwide study, we found a higher prevalence of comorbidity in patients with an advanced stage compared with a less advanced stage of ovarian cancer. One- and five-year mortality were nearly twice as high in patients with severe comorbidity compared to those without registered comorbidity, even after adjustment for stage. Thus the increased prevalence of more advanced stage did not entirely explain the association between greater comorbidity and higher mortality. We found further that the impact of severe comorbidity on one- and five-year mortality varied by cancer stage.

Among the strengths of our study were its large size and Denmark's uniformly organized health care system, allowing a population-based design with virtually complete follow-up. However, the accuracy of our findings depends on the quality of cancer registry and hospital discharge data. The Danish Cancer Registry is known to be more than 95\% complete [14], and in a previous study in which pathology records were used to confirm diagnoses, we found that ovarian cancer diagnoses were correct in $97 \%$ of the cases in the Registry, [30]. This minimal selection bias should not be related to the presence of comorbidity, however, since comorbidity was independently recorded before the cancer diagnosis.

Cancer staging is based on a combination of pathologic, operative, and clinical assessments available at the time of diagnosis. Some misclassification of stage data may occur, which could result in residual confounding. There may also have been some inaccuracy in treatment data used in the study. These data, obtained from the Danish Cancer Registry, are limited to treatment given within the first four months following diagnosis; since Registry reporting forms are often completed in the early period of treatment planning, they may not reflect actual treatment. If complications stemming from comorbidity lead to changes in treatment, this could result in residual confounding.

We used the validated Charlson Comorbidity Index as a measure of comorbidity. When applied to administrative data information on comorbidity is based on ICD-codes. The comorbid diseases may be coded with different accuracy in the different administrative registries and misclassifications occur in most registries [31]. The Charlson index has been shown to have a high specificity [4], but a more variable sensitivity when compared with diagnoses abstracted from the medical charts [32]. It is thus possible that some patients with comorbid conditions may have been classified erroneously as having Charlson score 0 . Similarly, patients with severe comorbidity may have been classified erroneously as having Charlson score 1-2. However, because comorbidity was independently recorded before the cancer diagnosis, any misclassifica- tion of comorbid conditions was probably unrelated to ovarian cancer stage.

Patients with comorbidities may experience a delay in diagnosis or they may actually be diagnosed earlier because they have a close relationship with the health care system. We found that the presence of severe comorbidity was associated with an advanced stage of ovarian cancer. If ovarian cancer progresses from Figo-stage I to IV this could suggest a delay in diagnosis. It has, however, been suggested that stage I and stage III may be different forms of the disease [33].

Our findings disagree with a Dutch population-based study by Mass et al. [6], which was restricted to approximately 500 patients with FIGO-stage II and III ovarian cancer. Using a slightly modified Charlson Comorbidity Index and adjusting for treatment, age, stage, and period of diagnosis, they concluded that comorbidity did not influence prognosis. Other cohort studies have had similar findings $[8,10,11]$. A Norwegian population-based cohort study $(\mathrm{N}=571)$ examining the impact of several possible prognostic factors on survival found that comorbidity was a prognostic factor in univariate but not multivariate analyses [11]. One reason this study did not find an association may be its adjustment for residual tumour. Presence of a residual tumour is related to the aggressiveness of surgery and if comorbidity results in less aggressive surgery, residual tumour may be an intermediate in the causal pathway from comorbidity to death. In this situation, adjustment for residual tumour would be inappropriate. The effect of comorbidity on mortality may be mediated to a large degree by higher volume of residual tumour. A Dutch population-based study $(\mathrm{N}=1,116)$ that adjusted for age, stage and treatment also did not find an independent effect of comorbidity on prognosis [10]. Similarly, an American hospital-based study reported an age-, stage- and symptom stage-adjusted mortality rate ratio of 1.04 in ovarian cancer patients with comorbidity compared to those with no comorbidity [8]. Its study population consisted of 137 ovarian cancer patients recruited during a period of almost 6 years, which could have introduced selection bias [8].

In accordance with our study, a negative impact of comorbidity on ovarian cancer mortality was found in an American population-based and in a German cohort study $[7,9]$. In these studies the mortality rate ratios were adjusted for stage, but the impact of stage on survival was not reported. The current study corroborates the findings in our recent study [12] on this topic. Our observation of the impact of comorbidity on mortality also confirm and extend findings for other groups of cancer patients, including breast cancer, prostate cancer, colon cancer, and lung cancer patients [34]. 
Since stage did not entirely account for the differences in mortality in our study, other factors may explain the role of comorbidity as a negative prognostic factor. The presence of comorbidity in a cancer patient may influence treatment choices, which in turn affect prognosis and survival $[10,35]$. The optimal treatment of patients with ovarian cancer is surgery and chemotherapy, with regimens depending on stage [36]. In advanced stages it is important to optimally debulk the tumour, and while this extensive surgery can be performed safely in patients with comorbid conditions [3], not all such patients receive this treatment [6]. It also is possible that following established treatment guidelines is not the best strategy for patients with multiple comorbidities [37], either because they cannot tolerate the adjuvant chemotherapy necessary after surgery or because the drugs used to treat their comorbid diseases may interact with those in chemotherapy regimens. As well, the toxicity of chemotherapy may be exacerbated by the side effects of the drugs that are used to treat comorbidities [38]. While we were able to adjust for treatment using data from the Danish Cancer Registry, information was lacking on the aggressiveness of the surgery performed or use of modified chemotherapy regimens. Adjustment for treatment in the analyses did not diminish the impact of severe comorbidity on the oneyear MRR. However, despite adjustment for treatment, aggressiveness of treatment could vary in the comorbidity groups, as indicated a Norwegian study [11].

Because our study addressed all-cause rather than causespecific mortality, patients could have died from their comorbidity or other causes not related to ovarian cancer. This may also explain some of the higher mortality in patients with comorbidity. However, it is difficult to distinguish between the contributions to mortality from the ovarian cancer itself and that from cancer complications or comorbidities. An example is death from heart-disease vs. death due to chemotherapy-related aggravation of preexisting cardiac problems.

There is a need for clinicians to be aware of the possible presence of comorbidity in ovarian cancer patients in order to improve their treatment. Patients with regional spread/FIGO stage II and III ovarian cancer are a particularly important subgroup in this context.

\section{Conclusion}

In this population-based study of ovarian cancer patients, we found that the presence of severe comorbidity (Charlson score 3+) was associated with an advanced stage of cancer. Mortality was also higher among patients with comorbidities, but could not be entirely explained by the cancer stage.

\section{Competing interests}

The author(s) declare that they have no competing interests.

\section{Authors' contributions}

MST participated in the design of the study, analysis and interpretation of results and has been drafting the manuscript. CD participated in the design of the study, analysis and interpretation of data. LP participated in the analysis and interpretation of data. HTS participated in the design of the study, interpretation of results and revised the manuscript critically. MN participated in the design of the study, in the interpretation of results and revised the manuscript critically. All authors read and approved the final manuscript.

\section{Acknowledgements}

This study received support from the Western Danish Research Forum for Health Sciences, the Research Foundation of Northern Jutland, Research Initiative of Aarhus University Hospital, Ebba and Aksel Schølin Foundation, Peder Kristian Tøftings and Dagmar Tøftings Foundation, Heinrich Kopps Foundation, Herta Christensens Foundation, Karen Elise Jensen Foundation, Institute of Clinical Medicine (Aarhus University), and the Danish Cancer Society. The financial supporters had no role in the design, conduct, analysis, or reporting of this study.

\section{References}

I. Sant M, Aareleid T, Berrino F, Bielska LM, Carli PM, Faivre J, Grosclaude P, Hedelin G, Matsuda T, Moller H, Moller T, Verdecchia A, Capocaccia R, Gatta G, Micheli A, Santaquilani M, Roazzi P, Lisi D: EUROCARE-3: survival of cancer patients diagnosed 199094--results and commentary. Ann Oncol 2003, I4 Suppl 5:v61-II8.

2. Janssen-Heijnen ML, Houterman S, Lemmens VE, Louwman MW, Coebergh JW: Age and co-morbidity in cancer patients: a population-based approach. Cancer Treat Res 2005, I 24:89-I07.

3. Sharma S, Driscoll D, Odunsi K, Venkatadri A, Lele S: Safety and efficacy of cytoreductive surgery for epithelial ovarian cancer in elderly and high-risk surgical patients. Am J Obstet Gynecol 2005, 193:2077-2082.

4. de Groot V, Beckerman H, Lankhorst GJ, Bouter LM: How to measure comorbidity. a critical review of available methods. J Clin Epidemiol 2003, 56:221-229.

5. West DW, Satariano WA, Ragland DR, Hiatt RA: Comorbidity and breast cancer survival: a comparison between black and white women. Ann Epidemiol 1996, 6:413-419.

6. Maas HA, Kruitwagen RF, Lemmens VE, Goey SH, Janssen-Heijnen $M L$ : The influence of age and co-morbidity on treatment and prognosis of ovarian cancer: a population-based study. Gynecol Oncol 2005, 97: 104-109.

7. du Bois A, Rochon J, Lamparter C, Pfisterer J: Pattern of care and impact of participation in clinical studies on the outcome in ovarian cancer. Int J Gynecol Cancer 2005, I 5:183-191.

8. DiSilvestro P, Peipert JF, Hogan JW, Granai CO: Prognostic value of clinical variables in ovarian cancer. J Clin Epidemiol 1997, 50:50I-505.

9. O'Malley CD, Cress RD, Campleman SL, Leiserowitz GS: Survival of Californian women with epithelial ovarian cancer, 19941996: a population-based study. Gynecol Oncol 2003, 91 :608-6I5.

10. Janssen-Heijnen ML, Houterman S, Lemmens VE, Louwman MW, Maas HA, Coebergh JW: Prognostic impact of increasing age and co-morbidity in cancer patients: a population-based approach. Crit Rev Oncol Hematol 2005, 55:231-240.

II. Tingulstad S, Skjeldestad FE, Halvorsen TB, Hagen B: Survival and prognostic factors in patients with ovarian cancer. Obstet Gynecol 2003, 101:885-891. 
12. Tetsche MS, Norgaard M, Jacobsen J, Wogelius P, Sorensen HT: Comorbidity and ovarian cancer survival in Denmark, 19952005: a population-based cohort study. Int J Gynecol Cancer 2007.

13. Marx $\mathrm{Cl}$, Moller $\mathrm{C}$, Bendixen A, Kehlet $\mathrm{H}$, Ottesen BS: [Ovarian cancer in Denmark. Status of the surgical intervention]. Ugeskr Laeger 2006, I68: 1537-1540.

14. Storm HH, Michelsen EV, Clemmensen IH, Pihl J: The Danish Cancer Registry--history, content, quality and use. Dan Med Bull 1997, 44:535-539.

15. oncology FIGO: Staging classifications and clinical practice guidelines of gynaecologic cancers. 2000 [http://www.figo.org/ docs/staging booklet.pdf].

16. Kjaerbye-Thygesen A, Huusom LD, Frederiksen K, Kjaer SK: Trends in the incidence and mortality of ovarian cancer in Denmark 1978-2002. Comparison with other Nordic countries. Acta Obstet Gynecol Scand 2005, 84: 1006-1012.

17. The Danish Cancer Registry. 2006. http://www.sundhed....registre/car.xls

18. National Broad of Health HS: Cancer Incidence in Denmark 1999 Copenhagen; 2003.

19. Andersen TF, Madsen M, Jorgensen J, Mellemkjoer L, Olsen JH: The Danish National Hospital Register. A valuable source of data for modern health sciences. Dan Med Bull 1999, 46:263-268.

20. Sundhedsstyrelsen: The National Board of Health. 2007. http:// www.sundheds....ister.aspx?lang $=\mathrm{da}$

21. Charlson ME, Pompei P, Ales KL, MacKenzie CR: A new method of classifying prognostic comorbidity in longitudinal studies: development and validation. J Chronic Dis 1987, 40:373-383.

22. Nuttall $M$, van der MJ, Emberton M: Charlson scores based on ICD-10 administrative data were valid in assessing comorbidity in patients undergoing urological cancer surgery. J Clin Epidemiol 2006, 59:265-273.

23. Rieker RJ, Hammer E, Eisele R, Schmid E, Hogel J: The impact of comorbidity on the overall survival and the cause of death in patients after colorectal cancer resection. Langenbecks Arch Surg 2002, 387:72-76.

24. Birim O, Kappetein AP, Bogers AJ: Charlson comorbidity index as a predictor of long-term outcome after surgery for nonsmall cell lung cancer. Eur J Cardiothorac Surg 2005, 28:759-762.

25. Newschaffer CJ, Bush TL, Penberthy LT: Comorbidity measurement in elderly female breast cancer patients with administrative and medical records data. J Clin Epidemiol 1997, 50:725-733.

26. Frank L: Epidemiology. When an entire country is a cohort. Science 2000, 287:2398-2399.

27. Kirkwood BR, Sterne JA: Medical Statistics 2 nd ed edition. Edited by: F. G and V. P. Oxford, Blackwell Publishing; 2003.

28. DR. C: Regression Models and Life-Tables. Journal of the Royal Statistical Society, Series B 1972, 34:187-220.

29. Rothman KJ: Epidemiology An Introduction 2nd edition. Oxford, Oxford University Press; 2002.

30. Tetsche MS, Nørgaard M, Skriver MV, Andersen ES, Lash TL, Sørensen HT: Accuracy of ovarian cancer ICD-10 diagnosis in a Danish population-based hospital discharge registry. Eur J Gynaecol Oncol 2005, 26:266-270.

31. Sørensen HT: Regional administrative health registries as a resource in clinical epidemiology. A study of options, strengths, limitations and data quality provided with example of use. Int J Risk Safety Med 1997, 10:1-22.

32. Wilchesky M, Tamblyn RM, Huang A: Validation of diagnostic codes within medical services claims. J Clin Epidemiol 2004, 57:|3|-|4|.

33. Yemelyanova AV, Cosin JA, Bidus MA, Boice CR, Seidman JD: Pathology of stage I versus stage III ovarian carcinoma with implications for pathogenesis and screening. Int J Gynecol Cancer 2007.

34. Read WL, Tierney RM, Page NC, Costas I, Govindan R, Spitznagel EL, Piccirillo JF: Differential prognostic impact of comorbidity. J Clin Oncol 2004, 22:3099-3103.

35. Nagel G, Wedding U, Hoyer H, Rohrig B, Katenkamp D: The impact of comorbidity on the survival of postmenopausal women with breast cancer. J Cancer Res Clin Oncol 2004.

36. Vasey PA, Herrstedt J, Jelic S: ESMO Minimum Clinical Recommendations for diagnosis, treatment and follow-up of epithelial ovarian carcinoma. Ann Oncol 2005, 16 Suppl I:il3-il5.
37. Gijsen R, Hoeymans N, Schellevis FG, Ruwaard D, Satariano WA, van den Bos GA: Causes and consequences of comorbidity: a review. J Clin Epidemiol 200I, 54:661-674.

38. Monfardini S: Prescribing anti-cancer drugs in elderly cancer patients. Eur J Cancer 2002, 38:234I-2346.

\section{Pre-publication history}

The pre-publication history for this paper can be accessed here:

\section{http://www.biomedcentral.com/1471-2407/8/31/prepub}

Publish with Biomed Central and every scientist can read your work free of charge

"BioMed Central will be the most significant development for disseminating the results of biomedical research in our lifetime. " Sir Paul Nurse, Cancer Research UK

Your research papers will be:

- available free of charge to the entire biomedical community

- peer reviewed and published immediately upon acceptance

- cited in PubMed and archived on PubMed Central

- yours - you keep the copyright

Submit your manuscript here:

http://www.biomedcentral.com/info/publishing_adv.asp
BioMedcentral 